\title{
Etnoteorias Parentais: Um Estudo com Mães Residentes no Interior e na Capital de Santa Catarina
}

\author{
Parental Ethnotheories: A Study with Mothers Living in the Capital \\ and Countryside of Santa Catarina State
}

\author{
Samira Mafioletti Macarini ${ }^{*}, a$, Gabriela Dal Forno Martins ${ }^{a}$, \\ Virgínia Azevedo Reis Sachetti ${ }^{b} \&$ Mauro Luís Vieira ${ }^{a}$ \\ ${ }^{a}$ Universidade Federal de Santa Catarina \\ ${ }^{b}$ Faculdade Metropolitana de Guaramirim
}

\begin{abstract}
Resumo
O estudo teve como objetivo investigar etnoteorias parentais em mães residentes na capital e interior de Santa Catarina. Foram aplicadas escalas de crenças sobre práticas de cuidado, metas de socialização e alocentrismo familiar em 50 mães de cada contexto cultural. Os resultados na capital indicaram predomínio de elementos autônomo-relacionais em práticas e alocentrismo familiar, enquanto que em termos de metas, as mães valorizaram a autonomia da criança. No interior, no que se refere a metas de socialização e alocentrismo familiar, foram identificados aspectos relacionais. Contudo, em termos de crenças sobre práticas de criação, constatou-se maior valorização da autonomia. Concluiu-se que ambos os contextos caracterizam-se por um modelo de self autônomo-relacional, embora com diferentes ênfases de acordo com a dimensão investigada.

Palavras-chave: Etnoteorias parentais; Crenças sobre práticas de cuidado; Metas de socialização; Alocentrismo familiar.

Abstract

The aim of this study was to investigate parental ethnotheories of mothers living in the capital city and in the countryside of Santa Catarina State. In each cultural environment, fifty mothers were interviewed based on scales that evaluated their beliefs about childrearing practices, socialization goals and degree of familism. Results from the capital demonstrated a predominance of autonomous-related elements for practices and familism, whereas in terms of socialization goals, mothers valued the autonomy of the child. In the countryside, relational aspects were identified for socialization goals and familism. However, for beliefs about childrearing practices it has been noted emphasis on autonomy. The conclusion was that both contexts are characterized by an autonomous-related self, although with different emphases according to the dimension investigated.

Keywords: Parental ethnotheories; Beliefs about childrearing practices; Socialization goals; Familism.
\end{abstract}

Várias pesquisas enfocando o comportamento em diferentes contextos e também as influências de crenças e valores sobre o comportamento humano, tanto no Brasil, como em outros países, demonstram a preocupação atual em superar visões tradicionais e mecanicistas do desenvolvimento humano que não considerem a relação existente entre contexto e comportamento (Keller, Borke, Yovsi, Lohaus \& Jensen, 2005; Keller et al., 2004; Keller et al., 2006; Lordelo, 2002; Lordelo, Fonseca, \& Araújo, 2000). Nesse sentido, estudos sobre valores, crenças, idéias e práticas parentais têm sido realizados por alguns autores, embora no Brasil ainda sejam escassos os estudos que investigam esses fenômenos em diferentes con-

${ }^{*}$ Universidade Federal de Santa Catarina, Departamento de Psicologia, Campus Universitário, Trindade, Florianópolis, SC, CEP 88040-900. Tel.: + (48) 37218606.

E-mail: maurolvieira@gmail.com textos (Keller et al., 2005; Keller et al., 2006; Harkness \& Super, 1996, 2005; Seidl de Moura et al., 2004; Vieira et al., 2010).

O que mães e pais pensam e como eles agem em relação aos filhos têm forte conotação cultural e, por conseqüência, contextual. Nesse sentido, as crenças podem ser consideradas como resultado de um processo social/cultural e individual (Harkness \& Super, 1996; Harkness, Super, Vanna Axia, Palacios, \& Welles-Nyström, 2001), sendo, dessa forma, desenvolvidas em um contexto específico, em um determinado local e em um determinado tempo. Ainda, pode-se afirmar que as compreensões culturais que os pais apresentam são organizadas em categorias mais amplas de crenças, denominadas sistemas culturais de crenças parentais ou etnoteorias parentais, as quais são traduzidas em termos de ações relativas à criação de filhos que parecem exercer influência na saúde e desenvolvimento das crianças. 
As etnoteorias parentais podem ser caracterizadas como o conjunto organizado de idéias que estão implícitos na atividade cotidiana, julgamentos, escolhas e decisões dos cuidadores, agindo como modelos para suas práticas parentais (Harkness \& Super, 1996). Juntamente com o ambiente físico e social, bem como costumes e práticas de cuidado compartilhadas e estabelecidas cultural e historicamente, elas constituem o "Nicho de Desenvolvimento", proposto por Harkness e Super (1996).

Ao mesmo tempo, as etnoteorias inseridas no Nicho de Desenvolvimento são partes dos "Modelos Culturais", os quais são sistemas mais amplos que englobam uma série de idéias a respeito de temas diversos, organizadas e compartilhadas pelos membros de um grupo cultural, geralmente implícitas, vistas como verdadeiras e traduzidas em práticas (Cole, 1998; Harkness et al., 2001; Suizzo, 2002). Três modelos culturais de self, mais especificamente, têm sido identificados e estudados em função de seus impactos nos processos de socialização e desenvolvimento da criança.

$\mathrm{O}$ primeiro deles trata-se do modelo cultural independente, o qual enfatiza a construção do self como único e distinto, sendo priorizadas metas pessoais e focando-se nas necessidades e direitos do indivíduo. Nesse modelo predominam as dimensões de autonomia e separação, característicos de sociedades urbanas pós-industriais com alto nível de escolaridade (Kagitçibasi, 2007; Keller et al., 2005; Keller et al., 2004).

O segundo modelo, denominado interdependente, concebe o self como fundamentalmente conectado aos demais membros do grupo em que está inserido, sendo priorizadas metas grupais e a focalização de papéis sociais, deveres e obrigações. Nesse tipo de orientação predominam as dimensões de heteronomia e relação, características de ambientes rurais baseados em economia de subsistência (Kagitçibasi, 2007; Keller et al., 2005; Keller et al., 2004).

Por fim, o último modelo, posteriormente descrito na literatura, denomina-se autônomo-relacional (Kagitçibasi, 1996, 2005, 2007) e compreende características combinadas de ambos os modelos anteriores, englobando autonomia e relação, em que o self é definido como autônomo quanto a sua ação e relacional quanto à proximidade interpessoal. Ele é característico de famílias de classe média, urbana e escolarizada em sociedades tradicionalmente interdependentes.

Um componente dos modelos culturais de self que será investigado na presente pesquisa é o alocentrismo familiar. Este se caracteriza pelo grau de vinculação do adulto com sua família de origem, a qual engloba sentimentos de lealdade, reciprocidade e solidariedade com a mesma. Nesse sentido, é esperado que seja maior em modelos culturais mais interdependentes, intermediário em contextos autônomo-relacionais e menor em contextos mais independentes (Keller et al., 2006; Lay et al., 1998).
Os cuidadores como membros de sua própria cultura compartilham modelos culturais de parentalidade, ou seja, possuem concepções comuns acerca das etnoteorias parentais de acordo com o contexto cultural em que estão inseridos. No presente estudo, as etnoteorias parentais serão descritas em termos de crenças sobre práticas de cuidado e de metas de socialização.

As crenças parentais sobre práticas de cuidado caracterizam-se pelo conjunto organizado de idéias que estão implícitas na atividade cotidiana e nos julgamentos, escolhas e decisões que os cuidadores tomam em relação a seus filhos (Harkness \& Super, 1996). Diversos estudos têm como objetivo investigar especificamente quais práticas são mais e menos valorizadas pelos pais de diferentes culturas, classe social, nível de escolaridade, entre outros. Existem evidências na literatura demonstrando que as crenças parentais sobre práticas de cuidado variam entre os diferentes contextos culturais (Harkness \& Super, 2005; Keller et al., 2005; Keller et al., 2006; Suizzo, 2002).

Keller et al. (2006), por exemplo, estudaram a parentalidade - em termos de crenças sobre práticas e metas de socialização - bem como aspectos do contexto cultural, através do alocentrismo familiar, em três distintos modelos culturais: independente, interdependente e autônomo-relacional. Os autores verificaram diferenças significativas nos grupos. Os participantes provenientes do modelo cultural independente obtiveram menores médias no que se refere ao alocentrismo familiar, bem como à dimensão relacional dos instrumentos de metas e práticas. Ao mesmo tempo, os participantes do grupo cultural interdependente apresentaram escores significativamente maiores nas três escalas, enquanto que os provenientes do grupo autônomo-relacional ocuparam uma posição intermediária.

Outro estudo importante acerca das crenças sobre práticas de cuidado foi realizado por Suizzo (2002), a qual estudou os modelos culturais de parentalidade entre pais e mães parisienses. O estudo identificou a existência de três dimensões de crenças: "estimulação", "apresentação apropriada da criança em público" e "responsividade". As duas primeiras foram as mais valorizadas, sendo a última pouco valorizada. Ainda, Suizzo (2002) verificou que as variáveis número de filhos e escolaridade foram positivamente associadas à dimensão "estimulação" e que a dimensão "apresentação apropriada da criança" foi positivamente associada ao nível de religiosidade, e negativamente associada à idade e à escolaridade.

Alguns estudos realizados no Brasil foram desenvolvidos com o objetivo de verificar as dimensões de crenças sobre práticas identificadas por Suizzo (Kobarg, 2006; Piovanotti, 2007; Ruela, 2006). Resultados dessas pesquisas demonstraram que as mães urbanas de alta escolaridade valorizam principalmente a dimensão de práticas de estimulação, caracterizada por proporcionar à criança diferentes tipos de estimulações, tais como 
Macarini, S. M., Martins, G. D. F., Sachetti, V. A. R. \& Vieira, M. L. (2010). Etnoteorias Parentais: Um Estudo com Mães Residentes no Interior e na Capital de Santa Catarina.

interações diádicas e grupais, estimulação com brinquedos, através da linguagem, cognição, entre outros. Já as mães com pouca escolaridade e também aquelas do interior, parecem valorizar mais a categoria de práticas de apresentação apropriada da criança em público, como o bom comportamento e higiene da criança.

Em um estudo de metodologia semelhante, porém de âmbito nacional, envolvendo sete capitais brasileiras, os resultados demonstraram a valorização tanto de práticas de estimulação quanto de apresentação apropriada, sendo a dimensão de responsividade a menos valorizada pelas mães (Vieira et al., 2010). Os autores ainda concluem que as dimensões de práticas parentais são compartilhadas por mães de diferentes regiões geográficas do país, e que as mesmas são moduladas pelo nível educacional e as condições culturais das mães.

Além das crenças sobre práticas de cuidado, outra dimensão importante que vem sendo pesquisada são as metas de socialização, as quais são caracterizadas pelo que os cuidadores desejam e valorizam para o futuro de seus filhos em termos de consequiências desenvolvimentais (Darling \& Steinberg, 1993). Alguns estudos têm investigado as metas de socialização utilizando uma metodologia de entrevista semi-estruturada de Miller e Harwood (2001), sendo verificadas diferentes valorizações das metas por pais residentes em contextos culturais distintos (Harwood, Schoelmerich, Schulze, \& Gozalez, 1999; Leyendecker, Harwood, Lamb, \& Sholmerich, 2002; Miller \& Harwood, 2001).

Os resultados desses estudos demonstram que as mães residentes em contextos mais independentes enfatizam metas relacionadas a auto-aperfeiçoamento e auto-controle da criança, visando o desenvolvimento da independência e da autonomia através do aumento da auto-estima e autoconfiança dos filhos. Já as mães residentes em contextos mais interdependentes valorizam o bom comportamento da criança e sua adequação às expectativas sociais, com ênfase no respeito, cooperação dentro do contexto social, conduta apropriada, relações interpessoais adequadas, capacidade de se entender com os outros e o cumprimento de todas as obrigações, particularmente dentro da família.

No Brasil, alguns estudos em que os autores utilizaram metodologia semelhante foram realizados para investigar as dimensões de metas existentes em diferentes contextos do país. Ruela (2006), por exemplo, comparou as metas das mães e avós de uma comunidade rural no Rio de Janeiro, verificando que as metas das mães concentraram-se nas categorias "auto-aperfeiçoamento" e "expectativas sociais", respectivamente, enquanto entre as avós houve um predomínio das categorias "bom comportamento, auto-aperfeiçoamento e expectativas sociais". Já os resultados de Piovanotti (2007), com mães urbanas da capital de Santa Catarina, indicaram que as categorias "expectativas sociais" e "auto-aperfeiçoamento", respectivamente, fazem parte do sistema de crenças da maioria absoluta da amostra pesquisada, não sendo verificadas influências das variáveis sociodemográficas.

Por fim, resultados semelhantes foram verificados por Moinhos, Lordelo e Seidl-de-Moura (2007) em mães de diferentes contextos socioeconômicos residentes da capital da Bahia. As metas mais mencionadas pelas mães foram respectivamente: "expectativas sociais", "autoaperfeiçoamento" e "bom comportamento", não havendo diferenças significativas quanto ao nível socioeconômico. Assim como Piovanotti (2007), as autoras mencionadas anteriormente afirmam que a não existência de diferenças poderia indicar uma maior homogeneidade de crenças e ideologias sustentadas por mães que compartilham do mesmo contexto cultural em comparação às práticas, que parecem ser mais afetadas pelas condições de vida presentes no contexto mais imediato.

A partir dos estudos apresentados é possível caracterizar contextos diversos em função dos modelos culturais predominantes. Tal caracterização pode ser realizada com base na avaliação de diferentes aspectos das etnoteorias parentais, assim como a partir da vinculação dos adultos com sua família de origem. Além disso, é importante investigar a relação existente entre estas dimensões, bem como suas diferentes configurações de acordo com o contexto sociocultural proximal em que se inserem. Dessa forma, acredita-se que o delineamento de estudos no Brasil possa trazer contribuições à literatura existente, complementando os resultados de estudos já realizados nos seus diferentes contextos.

A presente pesquisa teve como objetivo investigar dimensões de etnoteorias parentais - em termos de crenças sobre práticas de cuidado durante o primeiro ano de vida da criança e metas de socialização até os três primeiros anos de vida da mesma - bem como o grau de alocentrismo familiar, em mães residentes em dois contextos culturais distintos: capital e interior de um estado do sul do Brasil. Além disso, um segundo objetivo do estudo consiste na busca de relações entre as três dimensões investigadas com variáveis sociodemográficas, visando verificar a influência de características do contexto proximal nas etnoteorias que as mães possuem, bem como na vinculação com suas famílias de origem.

\section{Método}

\section{Participantes}

Participaram do estudo 100 mães residentes em dois contextos catarinenses distintos (50 na capital e 50 no interior), com idade acima de 21 anos, com pelo menos um filho entre 0 e 6 anos de idade na época da coleta de dados.

As 50 mães da capital Florianópolis apresentaram idade variando entre 21 e 49 anos, com média de 32,14 $(D P=6,37)$. A maioria delas possuía apenas um filho $(n=30)$, era casada ou vivia em união estável $(n=42)$, tinha um trabalho remunerado $(n=39)$ e foi criada na zona 
urbana ( $n=42)$. Nesse contexto, aproximadamente metade das mães $(n=26)$ eram católicas, 13 afirmaram não possuir religião e o restante era de outras religiões. Já as 50 mães residentes no interior apresentaram idade variando entre 21 e 38 anos, com média de 29,16 $(D P=3,82)$. A maioria delas também tinha apenas um filho $(n=28)$, era casada ou vivia em união estável $(n=46)$, possuía um trabalho remunerado $(n=43)$ e foi criada na zona rural $(n=28)$. Com relação à religião, a maior parte delas era católica $(n=33)$, sendo quinze de outras religiões e apenas duas não possuíam religião.

\section{Contextos}

A capital, Florianópolis, localizada no litoral, é uma cidade de colonização açoriana, que possui população estimada em 2006 de 406.564 habitantes distribuídos em uma área de $463,5 \mathrm{~km}^{2}$. A principal atividade econômica está ligada ao turismo e comércio. Em termos gerais de infra-estrutura, a cidade conta com 354 estabelecimentos de saúde, sendo 73 públicos, 3.784 creches/ centros de educação infantil freqüentadas por 176.316 crianças de 0 a 6 anos de idade, universidades, bibliotecas e áreas culturais e de lazer (Governo do Estado de Santa Catarina, 2006; Instituto Brasileiro de Geografia e Estatística [IBGE], 2006).

O município de Corupá localiza-se no planalto norte de Santa Catarina, a $63 \mathrm{~km}$ de Joinville e a $210 \mathrm{~km}$ de Florianópolis. É uma cidade de colonização predominantemente alemã e possui população estimada de 12.760 habitantes em 2005, distribuídos em uma área de aproximadamente $405 \mathrm{~km}^{2}$. Segundo dados do Governo do Estado de Santa Catarina (2006), o comércio e a indústria são responsáveis por mais da metade da renda do município, que é o maior produtor de banana do Estado. Em termos de infra-estrutura, a cidade conta com seis estabelecimentos de saúde para atendimento ambulatorial e 46 leitos hospitalares. Em 2005 havia 394 crianças matriculadas na educação infantil (IBGE, 2006).

O município de Schroeder possui 11. 378 habitantes distribuídos em aproximadamente $144 \mathrm{~km}^{2}$ (IBGE, 2006), está localizado no Vale do Itapocú, na micro-região de Joinville, a 187 km de Florianópolis e é de colonização alemã. A economia do município gira em torno do cultivo e industrialização da banana e de vários doces de frutas, além de fábricas de malha de algodão e agropecuária (Governo do Estado de Santa Catarina, 2006). Em termos de infra-estrutura, a cidade conta com quatro estabelecimentos de saúde para atendimento ambulatorial, sem leitos hospitalares. Em 2005 havia 197 crianças matriculadas na educação infantil (IBGE, 2006).

As duas últimas cidades foram agrupadas para fins de coleta de dados em um único contexto (interior) por serem semelhantes em vários aspectos: localização (norte do Estado de Santa Catarina com acesso pela rodovia BR 280), distância da capital, população, índice de escolarização, colonização basicamente alemã e principal atividade econômica ligada à produção de banana.

\section{Instrumentos}

As três escalas utilizadas no presente estudo foram traduzidas e adaptadas para o português pelo núcleo de pesquisa "Interação social e desenvolvimento" coordenado pela Profa. Maria Lúcia Seidl de Moura, do Instituto de Psicologia da Universidade Estadual do Rio de Janeiro, e validadas por Sachetti (2007). Essas escalas foram as seguintes:

Escala de Crenças sobre Práticas Parentais (Keller et al., 2006). Consiste de uma escala tipo Likert de cinco pontos. A escala varia de 1 (concordo nem um pouco) até 5 (concordo completamente), sendo que quanto mais próximo de cinco mais a mãe valoriza as práticas de cuidado constantes no instrumento. A versão brasileira da escala conte oito afirmativas para investigar crenças sobre a maneira de cuidar de bebês ou crianças pequenas de até um ano de idade. A escala é formada por duas dimensões: (a) práticas relacionais $(\alpha=0,65)$, a qual contém itens que enfatizam o contato corporal e satisfação imediata das necessidades físicas (por exemplo, se é importante embalar um bebê que chora para consolá-lo); e (b) práticas autônomas $(\alpha=0,49)$, a qual contém itens que focam a auto-regulação precoce da criança, estimulação por objetos e interação face-a-face (por exemplo, é bom para um bebê dormir sozinho).

Escala de Metas de Socialização (Keller et al., 2006). É também uma escala tipo Likert de cinco pontos, que varia de 1 (concordo nem um pouco) até 5 (concordo completamente). Quanto mais próximo de cinco mais a mãe valoriza as metas de socialização constantes no instrumento. A versão brasileira é formada por oito afirmativas para investigar crenças sobre o que uma criança deveria aprender ou desenvolver durantes os três primeiros anos de idade. A escala também é composta por duas dimensões: (a) metas relacionais $(\alpha=0,72)$, a qual inclui itens como "aprender a obedecer a pessoas mais velhas" e "aprender a animar os outros"; e (b) metas autônomas $(\alpha=0,76)$, a qual inclui itens como "desenvolver autoconfiança" e "desenvolver um senso de auto-estima".

Escala de Alocentrismo Familiar (Lay et al., 1998). Consiste de uma escala tipo Likert de cinco pontos, que varia de 1 (concordo nem um pouco) até 5 (concordo completamente). Quanto mais próximo de cinco, mais fortemente será a ênfase nas dimensões que compõem o instrumento. Na versão brasileira, a escala contem 14 itens referentes à dimensão alocêntrica $(\alpha=0,80)$, e 6 itens ligados ao idiocentrismo $(\alpha=0,56)$. Alocentrismo e idiocentrismo são dimensões do conceito que uma pessoa tem de si mesma, do ponto de vista da psicologia cultural e refletem dependência ou separação da família ou do grupo social mais próximo, respectivamente, e são úteis para investigar variações culturais. A dimensão alocentrismo-idiocentrismo foi empregada pelos autores que elaboraram a escala com o significado de conexão ao grupo de origem.

Além das escalas, também foi utilizado questionário de dados sociodemográficos visando investigar a idade 
Macarini, S. M., Martins, G. D. F., Sachetti, V. A. R. \& Vieira, M. L. (2010). Etnoteorias Parentais: Um Estudo com Mães Residentes no Interior e na Capital de Santa Catarina.

das mães, número de filhos, local de residência, configuração da família, escolaridade e renda familiar mensal.

\section{Procedimentos de Coleta de Dados}

Após aprovação pelo Comitê de Ética da Universidade Federal de Santa Catarina (UFSC) sob os $\mathrm{n}^{\text {os }}$. 166/06 (mães da capital) e 239/06 (mães do interior) iniciou-se a coleta de dados. O procedimento foi realizado por entrevistadoras, que seguiram as instruções constantes no Manual de Procedimentos para Coleta de Dados, o qual continha informações de como recrutar mães para participar da pesquisa, como realizar o primeiro contato, como manejar a entrevista, etc.

Nesta pesquisa, as mães foram recrutadas através de creches, postos de saúde, bem como indicações de outras participantes. A aplicação dos instrumentos foi individual. Em primeiro lugar, foram dadas informações sobre a pesquisa para as participantes. Após isso, as questões dos instrumentos eram lidas por uma entrevistadora que também registrava as respostas, enquanto a mãe acompanhava a leitura em uma outra via impressa dos instrumentos. As entrevistas tiveram duração aproximada de uma a duas horas e ao final as participantes ganharam um presente, procedimento usual em coleta de dados longa com pais, utilizado também em Keller et al. (2004).

\section{Análise dos Dados}

Os dados foram analisados através de análises descritivas, como cálculos de frequiência, média e porcenta- gens. Além disso, para comparar as médias nas escalas entre as mães residentes nos dois contextos e relacionar tais médias às variáveis sociodemográficas, foram realizados cálculos de comparação de médias para grupos independentes (teste $t$ ) e correlações (Pearson).

\section{Resultados}

\section{Caracterização Sociodemográfica das Mães da Capital e Interior}

Inicialmente foi realizada uma comparação entre as características sociodemográficas das mães residentes nos dois contextos estudados. Foram verificadas diferenças significativas na idade e escolaridade, indicando que as mais jovens estão no interior $(t(98)=0,298 ; p<0,01)$, enquanto que aquelas de maior escolaridade estão na capital $(t(98)=3,60 ; p<0,001)$.

Além disso, existe associação entre o contexto onde as mães residem e local de criação das mesmas: mães da capital foram criadas predominantemente na zona urbana e mães do interior foram criadas predominantemente na zona rural $\left(\chi^{2}=17,36 ; g=1 ; p<0,01\right)$. Esse dado reforça a existência de grupos bastante distintos, uma vez que as mães residentes nos dois contextos estudados, capital e interior, também nasceram nesses respectivos locais. Ainda, há indicações da ausência de imigração dessas mães, variável que poderia interferir nos principais resultados da pesquisa.

O resumo das principais características sociodemográficas das participantes está descrito na Tabela 1.

Tabela 1

Principais Características Sociodemográficas das Participantes

\begin{tabular}{lcc}
\hline Variáveis sócio-demográficas & Capital & Interior \\
& $M(D P)$ & $M(D P)$ \\
\hline Idade das mães* & $32,14 \pm 6,37$ & $29,16 \pm 3,82$ \\
Escolaridade das mães* A & $7,2 \pm 2,09$ & $5,8 \pm 1,78$ \\
Número de filhos & $1,5 \pm 0,7$ & $1,5 \pm 0,73$ \\
Número de pessoas que moram na casa & $3,6 \pm 0,88$ & $3,7 \pm 0,9$ \\
Renda familiar mensal $^{\mathrm{B}}$ & $11,88 \pm 2,46$ & $11,46 \pm 12,81$
\end{tabular}

Nota. * Diferença significativa $p<0,01 .{ }^{\text {A }}$ Escolaridade medida através de uma escala de 9 pontos em que: $1=$ Não alfabetizado; 2=Fundamental incompleto (até 4 a . Série); 3 = Fundamental incompleto ( $5^{\mathrm{a}}$. $-8^{\mathrm{a}}$. Série); $4=$ Fundamental completo; $5=$ Ensino Médio incompleto; 6 = Ensino Médio completo; 7 = Superior incompleto; 8 = Superior completo; 9 = Pós-Graduação. ${ }^{\text {B }}$ Renda Familiar Mensal (em reais) medida através de uma escala de 14 pontos em que: $1=$ Até $100 ; 2=101$ a 200; $3=201$ a 300; $4=301$ a $400 ; 5=401$ a $500 ; 6=501$ a $600 ; 7=$ De 601 a $800 ; 8=801$ a $1000 ; 9=1001$ a $1300 ; 10=1301$ a $1600 ; 11=1601$ a $2000 ; 12=2001$ a $3000 ;$ $13=3001$ a 4000; $14=$ Acima de 4000 .

\section{Práticas Parentais, Metas de Socialização e Alocentrismo Familiar}

As médias de respostas das mães da capital e do interior referentes às dimensões das medidas estudadas encontramse na Tabela 2. Através da análise estatística foram identificadas diferenças significativas dentro de cada contexto e também entre os contextos. Com relação às práticas parentais, as mães do interior valorizaram significativamente mais a dimensão autônoma $(t(49)=5,59 ; p<0,01)$, enquanto que as mães da capital valorizaram igualmente práticas autônomas e relacionais $(t(49)=1,84 ; p>0,05)$. Comparando os dois contextos, as mães do interior apresentaram médias significativamente maiores para práticas autônomas do que as mães da capital $(t(98)=2,09 ; p<0,05)$. Já as práticas relacionais foram valorizadas igualmente por mães de ambos os contextos $(t(98)=0,89 ; p>0,05)$. 
Diferente dos resultados de práticas, os dados obtidos nas metas de socialização demonstraram que as mães da capital valorizaram significativamente mais a dimensão autônoma $(t(49)=5,02 ; p<0,01)$, enquanto que as mães do interior valorizavam igualmente metas autônomas e relacionais $(t(49)=1,17 ; p>0,05)$. Comparando os dois contextos, as mães do interior apresentaram médias significativamente maiores para metas relacionais do que as mães da capital $(t(98)=3,80 ; p<0,01)$. Já as metas autônomas foram valorizadas igualmente por mães de ambos os contextos $(t(98)=0,26 ; p>0,05)$.
Quanto ao alocentrismo familiar, entre as mães do interior verificou-se um predomínio significativo da dimensão alocêntrica $(t(49)=5,91 ; p<0,01)$, enquanto que na capital as mães apresentaram médias semelhantes para alocentrismo e idiocentrismo $(t(49)=0,81 ; p>0,05)$. Comparando os dois contextos, constatou-se que as mães do interior apresentaram médias significativamente maiores para o alocentrismo do que as mães da capital $(t(98)=3.78$; $p<0,01$ ), apontando que as primeiras são predominantemente mais vinculadas à família. Já em termos de idiocentrismo, não foi verificada diferença significativa entre as mães de ambos os contextos $(t(98)=0,61 ; p>0,05)$.

Tabela 2

Diferenças entre Interior e Capital: Comparação de Médias e Desvios Padrões de Práticas, Metas e Alocentrismo dentre e entre os Grupos

\begin{tabular}{|c|c|c|c|c|c|}
\hline & \multicolumn{2}{|c|}{ Interior } & \multicolumn{2}{|c|}{ Capital } & \multirow[b]{2}{*}{$t(98)$} \\
\hline & $M$ & $D P$ & $M$ & $D P$ & \\
\hline \multicolumn{6}{|c|}{ Práticas Parentais } \\
\hline relacional & 3,13 &, \pm 83 & 3,28 &, \pm 85 &, 89 \\
\hline autonomia & 3,86 &, \pm 68 & 3,54 &, \pm 86 & $2,09^{*}$ \\
\hline$t(49)$ & \multicolumn{2}{|c|}{$5,59 * *$} & \multicolumn{2}{|c|}{1,84} & \\
\hline \multicolumn{6}{|c|}{ Metas de Socialização } \\
\hline relacional & 3,81 &, \pm 74 & 3,21 &, \pm 84 & $3,80 * *$ \\
\hline autonomia & 3,94 &, \pm 80 & 3,90 &, \pm 72 & ,26 \\
\hline$t(49)$ & \multicolumn{2}{|c|}{1,17} & \multicolumn{2}{|c|}{$5,02 * *$} & \\
\hline \multicolumn{6}{|c|}{ Alocentrismo Familiar } \\
\hline alocêntrico & 3,80 &, \pm 61 & 3,37 &, \pm 53 & $3,78 * *$ \\
\hline idiocêntrico & 3,17 &, \pm 77 & 3,25 &, \pm 65 & ,61 \\
\hline$t(49)$ & \multicolumn{2}{|c|}{$5,91 * *$} & \multicolumn{2}{|c|}{0,81} & \\
\hline
\end{tabular}

Nota. $* p<0,05 ; * * p<0,01$.

Relação entre as Dimensões de Etnoteorias e Variáveis Sociodemográficas

Para verificar influências de variáveis do contexto sociodemográfico específico nas dimensões de práticas, metas e alocentrismo familiar, utilizou-se a análise de correlação com as seguintes variáveis: idade da mãe, escolaridade da mãe, número de filhos, número de pessoas na casa e renda familiar mensal. Estas variáveis foram selecionadas em função de sua influência na parentalidade, como a idade e escolaridade da mãe, número de filhos e renda familiar mensal. Outras variáveis como o número de pessoas na casa, por sua vez, tem relação com o número de filhos, bem como com o modelo teórico dos modelos culturais de autonomia e interdependência. Como apresentado na introdução, em contextos interdependentes, é esperado um maior número de filhos, mais proximidade nas relações interpessoais e, conseqüentemente, um maior número de pessoas residentes na casa.

A correlação das dimensões de práticas com dados sociodemográficos na capital demonstrou que a escola- ridade da mãe é uma variável significativa neste contexto. Verificou-se correlação negativa entre esta variável e as duas dimensões do instrumento, indicando que quanto maior a escolaridade menor a realização de práticas relacionais $(r=-0,29 ; p<0,05)$ e menor realização de práticas autônomas $(r=-0,35 ; p<0,01)$. Constatou-se também uma correlação significativa entre as dimensões da escala para as mães da capital: quanto mais realizam práticas relacionais, mais realizam práticas autônomas $(r=0,32 ; p<0,05)$, sugerindo a presença de elementos do modelo autônomo-relacional entre as mães urbanas. Já no interior, a correlação entre práticas e dados sóciodemográficos apontou que o número de filhos parece ser uma variável importante neste contexto, uma vez que mães com mais filhos realizam mais práticas relacionais $(r=0,31 ; p<0,05)$.

Com relação às metas, na capital, verificou-se que, assim como nas práticas, a escolaridade da mãe parece ser um fator bastante importante na modulação desse fenômeno. Além desta variável, a renda familiar mensal e o número de pessoas que vivem na casa também apre- 
Macarini, S. M., Martins, G. D. F., Sachetti, V. A. R. \& Vieira, M. L. (2010). Etnoteorias Parentais: Um Estudo com Mães Residentes no Interior e na Capital de Santa Catarina.

sentaram valores significativos de correlação. Verificouse que quanto maior a escolaridade $(r=-0,55 ; p<0,01) \mathrm{e}$ a renda $(r=-0,59 ; p<0,01)$, menores as médias para metas relacionais; e quanto mais pessoas vivem na casa, menores as médias para metas autônomas $(r=-0,34$; $p<0,01)$. Já no interior, a correlação das metas de socialização com dados sócio-demográficos demonstrou resultados significativos apenas para renda familiar mensal, indicando que quanto maior esta variável, menores as médias para metas autônomas $(r=-0,33 ; p<0,05)$. As dimensões da escala de metas também são correlacionadas entre si para a amostra residente no interior, indicando que quanto mais as mães valorizam metas autônomas, mais valorizam metas relacionais $(r=0,47 ; p<0,01)$. Esse dado referente às metas de socialização das mães do interior também sugere a presença de elementos do modelo autônomo-relacional nesse contexto.

Com relação às dimensões de alocentrismo familiar, não foi verificada nenhuma correlação significativa com as variáveis sociodemográficas selecionadas. Tal resultado parece indicar que o alocentrismo teria maior influência do contexto cultural específico do que de variáveis familiares mais próximas.

\section{Discussão}

A realização da presente pesquisa surgiu da verificação da necessidade de estudos que investiguem a parentalidade em diferentes modelos culturais, compostos de características sociodemográficas distintas. Para isso, buscou-se investigar crenças sobre práticas de mães durante o primeiro ano de vida da criança, as metas de socialização que as mesmas possuem a respeito dos filhos, bem como o grau de alocentrismo familiar, em dois contextos socioculturais sul-brasileiros. Dentre os principais resultados, verificou-se a presença de elementos autônomos e relacionais em ambos os locais estudados, indicando a predominância do modelo autônomo-relacional de self tanto na capital quanto no interior. Esse tipo de orientação cultural engloba autonomia e relação, em que o self é definido como autônomo quanto a sua ação e relacional quanto à proximidade interpessoal (Kagitçibasi, 1996, 2005, 2007). Além disso, para Kagitçibasi (2007), o modelo autônomo-relacional de self caracteriza-se como psicologicamente adequado, uma vez que envolve a satisfação de duas necessidades humanas: a existência de autonomia e relação.

Em termos de uma caracterização sociodemográfica, verificou-se que as mães residentes na capital possuíam em média apenas um filho, tinham significativamente mais idade e possuíam maior escolaridade que as mães do interior, dados que indicam a valorização do ensino e a postergação do nascimento do primeiro filho. Com relação à renda familiar mensal das mães, através da visualização dos valores de média e desvio padrão, podese verificar que a variação dos rendimentos das mães no interior foi maior que na capital, onde os valores foram mais homogêneos e, portanto, mais altos. Estas características sociodemográficas das mães da capital parecem indicar aspectos de um modelo cultural mais autônomo, descrito por Keller et al. (2006), o qual consiste de sociedades industrializadas, com alto grau de escolaridade, reprodução tardia e baixo número de filhos.

No que se refere às metas de socialização que as mães da capital possuem a respeito de seus filhos, também foram encontrados aspectos mais autônomos, uma vez que as mães valorizaram significativamente mais as metas autônomas do que as relacionais. Pode-se inferir que esse desejo das mães que seus filhos desenvolvam cada vez mais cedo independência, auto-confiança, senso de identidade e auto-estima, esteja relacionada com algumas transformações que ocorreram na sociedade brasileira nos últimos anos, principalmente nas capital e contextos mais urbanos. Dentre essas mudanças, Dessen e Torres (2002) citam a globalização e a modernização, os novos padrões de consumo e a flexibilidade no mundo do trabalho. Para essas autoras, tais fatores macro-sociais e históricos possivelmente modificaram as características das famílias brasileiras e consequentemente as idéias acerca do desenvolvimento da criança e a maneira de criar os filhos. Biasoli-Alves (2002) cita ainda alguns aspectos mais específicos como o aumento da escolarização; a entrada cada vez mais precoce das crianças na escola; as alterações dos papéis femininos e masculinos; a disseminação dos conhecimentos científicos como base para a orientação das relações interpessoais.

Nesse mesmo sentido, a correlação das dimensões estudadas com dados sociodemográficos na capital indicou que quanto maior a escolarização e renda familiar das mães, e quanto mais baixo o número de pessoas residentes no lar, maior o predomínio da dimensão de metas autônomas. Outros estudos, realizados dentro e fora do Brasil, encontraram resultados semelhantes, principalmente no que se refere ao nível de escolaridade das mães, que é considerada variável chave para o estudo da parentalidade (Goodnow, 1988; Kobarg, 2006; Lordelo et al., 2000; Piovanotti, 2007; Seidl de Moura et al., 2004; Suizzo, 2002). No caso do Brasil, o aumento da escolarização é um fator que parece exercer grande influência na estrutura do cotidiano das pessoas, trazendo reflexos também na forma de criar e educar os filhos (BiasoliAlves, 2002). Segundo dados do IBGE (2000), entre os anos de 1991 e 2000, houve um aumento de $17,2 \%$ da população acima de 25 anos de idade que possuem curso superior completo (graduação, mestrado ou doutorado). Ainda, entre 1940 e 2000, verificou-se um aumento de $45 \%$ da população alfabetizada, ou seja, que sabe ler e escrever (IBGE, 2007).

Ainda na capital, no entanto, em termos de práticas e alocentrismo familiar, foram verificados elementos do modelo autônomo-relacional. As mães valorizaram igualmente as práticas autônomas e as relacionais, ou seja, atribuíram igual importância à realização de práticas que visem tanto o desenvolvimento da independência desde 
cedo na criança, quanto da interdependência e autoregulação. Além disso, o grau de alocentrismo e idiocentrismo também não diferiu, o que indica que, ao mesmo tempo em que elas estão ligadas a sua família de origem, também conseguem manter uma certa distância e individualização. Esses dados se assemelham com os encontrados por Keller et al. (2006) em contextos autônomorelacionais como Beijing e Taiwan (China), São José (Costa Rica), Cidade do México (México) e Delhi (Índia). Os autores verificaram que tais contextos ocuparam uma posição intermediária em relação a contextos autônomos, de um lado, e relacionais de outro. Além disso, em geral, nestes contextos também foram encontradas médias bastante semelhantes para as dimensões autônomo e relacional de cada instrumento investigado (Keller et al., 2006).

No interior, em termos de uma caracterização sóciodemográfica, verificou-se menor idade e escolaridade das mães, com uma maior variação da renda familiar mensal. Apesar de serem mais novas, estas mães não apresentaram número de filhos mais elevado que as da capital. Esperava-se, ao contrário, que elas tivessem mais filhos, o que indicaria uma estratégia reprodutiva quantitativa, característica de sociedades mais interdependentes, de acordo com Keller et al. (2006). Uma outra característica das mães do interior refere-se a sua origem étnica alemã, a qual é descrita na literatura como predominantemente autônoma e independente (Keller et al., 2005; Keller et al., 2004). Estes dados sociodemográficos das mães do interior, sendo elas mais novas, de escolaridade moderada, de origem étnica alemã, com formação mais precoce de uma família e do nascimento do primeiro filho e estratégia reprodutiva não tanto quantitativa, parecem indicar características tanto autônomas quanto relacionais, o que resultaria em um modelo mais autônomo-relacional.

Esses dados corroboram com os resultados de práticas, metas e alocentrismo, uma vez que também foi verificada a presença de elementos tanto autônomos quanto relacionais através da investigação de tais dimensões no interior. Os dados obtidos em metas e alocentrismo evidenciaram a presença da dimensão relacional, enquanto os resultados de práticas indicaram aspectos mais autônomos nesse contexto. Em termos de metas, verificou-se que as mães valorizaram igualmente as dimensões autônomo e relacional, porém obtiveram médias significativamente maiores nas metas relacionais do que as mães da capital. Constatou-se também que as mães residentes no interior foram mais alocêntricas do que idiocêntricas, ou seja, apresentaram-se predominantemente vinculadas a sua família de origem. Esse valor também foi superior ao obtido entre as mães da capital. A partir desses dados, pode-se sugerir que o interior caracteriza-se por ser um contexto onde há mais proximidade física entre as pessoas, sendo que também se espera dos filhos que eles desenvolvam essa capacidade de se relacionar bem com os outros, respeitando os pais e pessoas mais velhas.
No entanto, apesar dessa proximidade com a família de origem e da valorização de habilidades relacionais nos filhos, em termos de práticas, as mães do interior valorizaram mais a dimensão autônoma, desejando que seus filhos sejam independentes desde cedo. Esse resultado não era esperado, uma vez que se imaginava que haveria um predomínio da dimensão relacional, assim como foi constatado em termos de metas e alocentrismo. Uma possível explicação para o que foi verificado seria o próprio conteúdo dos itens que compõem a dimensão de práticas autônomas, o qual está relacionado com rotinas (dormir a noite toda, por exemplo), além da habilidade da criança de realizar determinados comportamentos sozinha (dormir, interagir com objetos e brinquedos; por exemplo). Tais aspectos provavelmente foram mais valorizados em função das necessidades das mães, que trabalham tanto fora quanto dentro de casa e precisam que os filhos tenham rotinas e brinquem mais sozinhos desde cedo. Além disso, a origem étnica alemã das mães deste contexto também provavelmente influencia na valorização de práticas autônomas nos seus filhos.

Por outro lado, verificou-se que o número de filhos e a renda familiar parecem ser variáveis importantes na modulação do fenômeno da parentalidade no interior. Quanto maior o número de filhos, maior a valorização de práticas relacionais; além disso, quanto menos renda, mais metas autônomas. Pode-se pensar que quanto mais filhos, maior a necessidade das mães de garantir que os mesmos convivam em harmonia e proximidade, não sendo dada tanta ênfase a independência e a estimulação da autonomia de cada um dos filhos.

\section{Considerações Finais}

Através dos resultados encontrados nesse estudo entende-se que eles podem auxiliar no delineamento de um modelo cultural de parentalidade no contexto brasileiro. Além disso, os dados obtidos podem também complementar a compreensão que a literatura já possui sobre como os pais criam seus filhos, que qualidades esperam que os mesmos desenvolvam ao longo do seu crescimento e a relação disso com o grau de ligação dos pais com sua família de origem. Um resultado relevante é a indicação de que o contexto sul-brasileiro, mais especificamente o estado de Santa Catarina, caracteriza-se pelo predomínio de um modelo cultural autônomo-relacional, porém com diferenças e similaridades bastante significativas entre interior e capital.

É importante também que fiquem evidenciadas algumas limitações da presente pesquisa, bem como sugestões de outros estudos na área. Em relação ao método, sugerem-se estudos que utilizem outras formas de abordagem às mães para investigação das dimensões de práticas e metas, como a entrevista aberta e a observação. É possível que, dessa forma, sejam acessadas outras variáveis que interferem no fenômeno da parentalidade, 
Macarini, S. M., Martins, G. D. F., Sachetti, V. A. R. \& Vieira, M. L. (2010). Etnoteorias Parentais: Um Estudo com Mães Residentes no Interior e na Capital de Santa Catarina.

bem como seja possível realizar uma caracterização mais aprofundada.

\section{Referências}

Biasoli-Alves, Z. M. (2002). A questão da disciplina na prática de educação da criança, no Brasil, ao longo do século XX. Veritat, 2(2), 243-259.

Cole, M. (1998). Cultural psychology: A once and future discipline. Cambridge, MA: Harvard University Press.

Darling, N., \& Steinberg, L. (1993). Parenting style as context: As integrative model. Psychological Bulletin, 13(3), 487496.

Dessen, M. A., \& Torres, C. V. (2002). Family and socialization factors in Brazil: An overview. In W. J. Lonner, D. L. Dinnel, S. A. Hayes, \& D. N. Sattler, Online readings in psychology and culture (chap. 2). Bellingham, WA: Western Washington University. Retrieved from http://www.wwu.edu/ culture

Goodnow, J. J. (1988). Parents ideas, actions, and feelings: Models and methods from developmental and social psychology. Child Development, 59, 286-320.

Governo do Estado de Santa Catarina. (2006). Municípios. Retrieved from http://www.sc.gov/conteudo/municipios

Harkness, S., \& Super, C. M. (1996). Introduction. In S. Harkness \& C. M. Super (Eds.), Parents' cultural beliefs systems: Their origins expressions, and consequences (pp. 1-23). New York: The Guilford Press.

Harkness, S., \& Super, C. M. (2005). Themes and variations: Parental ethnotheories in Western cultures. In K. H. Rubin, \& O. B. Chung, Parental beliefs, parenting, and child development in cross-cultural perspective (pp. 61-79). New York: Psychology Press.

Harkness, S., Super, C. M., Vanna Axia, A. E., Palacios, J., \& Welles-Nyström, B. (2001). Cultural pathways to successful parenting. International Society for the Study of Behavior Development Newsletter, 38, 9-11.

Harwood, R. L., Schoelmerich, A., Schulze, P. A., \& Gozalez, Z. (1999). Cultural differences in maternal beliefs and behaviors: A study of middle-class Anglo and Puerto Rican mother-infant pairs in four everyday situations. Child Development, 70, 805-816.

Instituto Brasileiro de Geografia e Estatística. (2000). Censo demográfico - 2000. Retrieved from http://www.ibge.gov.br

Instituto Brasileiro de Geografia e Estatística. (2006). Cidades. Retrieved from http://www.ibge.gov.br

Instituto Brasileiro de Geografia e Estatística. (2007). Estatísticas do século XX. Retrieved from http://www.ibge.gov.br

Kagitçibasi, Ç. (1996). The autonomous-relational self: A new synthesis. European Psychologist, 1(3), 180-186.

Kagitçibasi, Ç. (2005). Autonomy and relatedness in cultural context: Implications for self and family. Journal of CrossCultural Psychology, 36, 403-422.

Kagitçibasi, Ç. (2007). Family, self, and human development across cultures: Theory and applications ( $2 \mathrm{nd}$ ed.). Mahwah, NJ: Lawrence Erlbaum.

Keller, H., Borke, J., Yovsi, R., Lohaus, A., \& Jensen, H. (2005). Cultural orientations and historical changes as predictors of parenting behaviour. International Journal of Behavioral Development, 29(3), 229-237.

Keller, H., Hentschel, E., Yovsi, R. D., Lamm, B., Abels, M., \& Haas, V. (2004). The psycho-linguistic embodiment of parental ethnotheories: A new avenue to understanding cultural processes in parental reasoning. Culture and Psychology, 10(3), 293-330.
Keller, H., Lamm, B., Abels, M., Yovsi, R., Borke, J., Jensen, H., et al. (2006). Cultural models, socialization goals and parenting ethnotheories: A multicultural analysis. Journal of Cross-Cultural Psychology, 37(2), 155-172.

Kobarg, A. P. (2006). Crenças e práticas de mães sobre o desenvolvimento infantil nos contextos rural e urbano. Dissertação de Mestrado não-publicada, Universidade Federal de Santa Catarina, Florianópolis, SC.

Lay, C., Fairlie, P., Jackson, S., Ricci, T., Eisenberg, J., Sato, T., et al. (1998). Domain-specific allocentrism idiocentrism. Journal of Cross-Cultural Psychology, 29(3), 434-460.

Leyendecker, B., Harwood, R. L., Lamb, M. E., \& Sholmerich, A. (2002). Mothers' socialisation goals and evaluations of desirable and undesirable everyday situations in two diverse cultural groups. International Journal of Behavioral Development, 26(3), 248-258.

Lordelo, E. R. (2002). Interação social e responsividade em ambientes doméstico e de creche: Cultura e desenvolvimento. Estudos de Psicologia (Natal), 7(2), 343-350.

Lordelo, E., Fonseca, A., \& Araújo, M. (2000). Responsividade do ambiente de desenvolvimento: Crenças e práticas como sistema cultural de criação de filhos. Psicologia: Reflexão e Crítica, 13(1), 73-80.

Miller, S. A., \& Harwood, R. L. (2001). Long-term socialisation goals and the construction of infants' social networks among middle class Anglo and Puerto Rican mothers. International Journal of Behavioral Development, 25(5), 450-457.

Moinhos, M. V., Lordelo, E. R., \& Seidl-de-Moura, M. L. (2007). Metas de socialização de mães baianas de diferentes contextos socioeconômicos. Revista Brasileira de Crescimento e Desenvolvimento Humano, 17(1), 114-125.

Piovanotti, M. R. (2007). Metas de socialização infantil e crenças sobre práticas de cuidador parental de mães primíparas. Dissertação de Mestrado não-publicada, Universidade Federal de Santa Catarina, Florianópolis, SC.

Ruela, S. (2006). Um estudo intergeracional de crenças valorizadas por mães em uma comunidade rural do Estado do Rio de Janeiro. Dissertação de Mestrado não-publicada, Universidade Estadual do Rio de Janeiro, RJ.

Sachetti, V. A. R. (2007). Um estudo das crenças maternas sobre cuidados com crianças em dois contextos culturais do Estado de Santa Catarina. Tese de Doutorado nãopublicada, Universidade Federal de Santa Catarina, Florianópolis, SC.

Seidl de Moura, M. L., Ribas, R. C., Jr., Piccinini, C. A., Bastos, A. C., Magalhães, C. M., Vieira, M. L., et al. (2004). Conhecimento sobre desenvolvimento infantil em mães primíparas de diferentes centros urbanos do Brasil. Estudos de Psicologia (Natal), 9(3), 421-429.

Suizzo, M. A. (2002). French parents' cultural models and childrearing beliefs. International Journal of Behavioral Development, 26, 297-307.

Vieira, M. L., Seidl-de-Moura, M. L., Lordelo, E., Piccinnini, C. A., Martins, G. D., Macarini, S. M., et al. (2010). Brazilian mother's beliefs about childrearing practices. Journal of Cross-Cultural Psychology, 41(2), 195-211. 